\title{
Histologic Evaluation of Blood Vessels Sealed with 1,470-nm Diode Laser: Determination of Adequate Condition for Laser Vessel Sealing
}

\author{
Nu-Ri $I m^{1}$ \\ Jungho Moon ${ }^{2}$ \\ Wonshik Choi \\ Byoungjae $\mathrm{Kim}^{1,3}$ \\ Jung Joo Lee ${ }^{4}$ \\ Heejin $\mathrm{Kim}^{4}$ \\ Seung-Kuk Baek ${ }^{1}$
}

${ }^{1}$ Department of Otolaryngology-Head and Neck Surgery, Korea University College of Medicine, Seoul, Korea

${ }^{2}$ Department of Physics, Korea University, Seoul, Korea

${ }^{3}$ Department of Physiology, Korea University, Seoul, Korea

${ }^{4}$ LivsMed Inc. Seoul, Korea
Received June 21, 2018

Accepted June 22, 2018

Correspondence
Seung-Kuk Baek
Department of Otolaryngology-Head and Neck
Surgery, Korea University College of Medicine,
126-1 Anam-dong 5-ga, Seongbuk-gu, Seoul
02841, Korea
Tel.: +82-2-920-5486
Fax: +82-2-925-5233
E-mail: mdskbaek@agmail.com
(C) Korean Society for Laser Medicine and Surgery
@c This is an open access article distributed under the
terms of the Creative Commons Attribution Non-
Commercial License (http://creativecommons.org/
licenses/by-nc/4.0) which permits unrestricted non-
commercial use, distribution, and reproduction in any
medium, provided the original work is properly cited.

\author{
Introduction \\ Energy-based devices allow for a more rapid and efficient ligation of \\ blood vessels during operations. In the present study, we evaluated the \\ feasibility of a laser as an alternative energy source for the vessel sealing \\ system and determined the optimal condition of laser for an effective \\ vessel sealing through histologic examination.
}

\section{Materials and Methods}

The arteries ( $5 \mathrm{~mm}$ diameter) harvested from porcine legs were compressed between two glass-slides to eliminate its luminal space and were irradiated with 1,470-nm diode laser under various sealing conditions, including laser power (5-30 W), irradiation time (5 or 10 seconds), and focus mode (focus or defocus). Subsequently, the irradiated vessels were fixed in $4 \%$ formaldehyde and then processed to paraffin block. The paraffinized sample was sectioned and stained with hematoxylin and eosin for histological evaluation.

\section{Results}

The extent of tissue change was positively correlated with duration and power of laser. In defocus mode, the irradiated vessels showed sufficient tissue denaturation for sealing effect without severe tissue destruction. Moreover, among the various conditions of irradiation, laser power between 15 and $20 \mathrm{~W}$, as well as exposure time of 5 seconds were appropriate for sealing the blood vessels.

\section{Conclusion}

Adequate power and irradiation duration of laser can render blood vessels to be sealed effectively, although the higher power of laser may be required to cut the vessels.

\section{Key words}

Vessels; Sealing; Diode Laser 


\section{INTRODUCTION}

Suture ligation and hemostasis of blood vessels during surgery is a time-consuming and skill-intensive process. To reduce these burdens, several energy-based devices using ultrasound and radiofrequency (RF) have been used for blood vessel sealing system. ${ }^{1,2}$ Whereas ultrasonic devices make use of the thermal energy of rapid vibration for coagulation and cutting blood vessels, RF-based devices achieve hemostasis and coagulation with heat generated by electrical current. ${ }^{3}$ These energy-based devices can be rapid and efficient in blood vessel ligation, which reduce times and costs significantly in diverse surgical environment. ${ }^{4-6}$

However, each of the energy-based devices has some weaknesses. RF-based devices can make wider denaturation and collateral damage after blood vessel sealing compared to ultrasound devices. ${ }^{7,8}$ In contrast, although ultrasonic devices show less thermal spread than RF devices, the maximal temperature of those instrument tip increase over $200^{\circ} \mathrm{C}$, which takes over 20 seconds to cool to usable condition. ${ }^{9}$ In addition, ultrasonic devices may not be easy to be flexible due to their acting mechanism.

To overcome these drawbacks, new energy sources to be installed in surgical devices are needed. Since laser has unique characteristics such as beam focusing and flexibility, laser can be made as a flexible device, cut and heat a tissue precisely. Recently, optical based systems have been studied as the new surgical instrument for vessel sealing. The infrared lasers with diverse wavelengths from 808 to $1,908 \mathrm{~nm}$ were investigated for coagulation and sealing for wide range of blood vessel diameters and the high-power 1,470 nm diode laser showed higher burst pressure and less thermal spread than other energybased devices. ${ }^{10,11}$

However, although these preliminary studies have shown the possibility of 1,470 $\mathrm{nm}$ diode laser as an alternative device, further study for the exact condition of laser-based device is needed for making one-step cutting and rapid sealing device. Thus, this study aimed at histologic and functional investigation of various condition in laser power, irradiation time and focus/defocus mode for safe and efficient vessel sealing system using 1,470-nm diode laser.

\section{MATERIALS AND METHODS}

\section{Tissue preparation}

Porcine femoral and radial arteries were used for all laboratory studies. First of all, $40 \mathrm{~kg}$ female pigs were anaesthetized and euthanized with Zoletil $505 \mathrm{mg} / \mathrm{kg}$, Rompun $2 \mathrm{mg} / \mathrm{kg}$ and $\mathrm{KCl} 2 \mathrm{mmol} / \mathrm{kg}$. After obtained from front and hind legs, femoral and radial arteries with 5 to $6 \mathrm{~mm}$ diameter were processed by removal of fat and stored in phosphate buffered saline prior to use. Then arteries were cut into $2.5 \mathrm{~mm}$ length to fit laser irradiation setup.

\section{Experimental setup}

We designed a bench-top setup for testing the laser sealing and cutting of blood vessels (Fig. 1A). Using this system, we identified the optimal conditions in laser power, beam shape, and irradiation time for the effective laser surgery.

A diode laser with the wavelength of 1,470 nm (QPC laser; Laser Operation LLC, CA, USA) and output power of $45 \mathrm{~W}$ was coupled to the fiber with $400-\mu \mathrm{m}$-core diameter (People Laser Tech, Anyang, Korea). A spherical lens (People Laser Tech) with the focal length of $30 \mathrm{~mm}$ collimated the output beam from the fiber. A cylindrical lens (model LJ1212L1-C; Thorlabs, NJ, USA) with focal length of $100 \mathrm{~mm}$ was used to focus one axis of the beam such that a line beam was generated at the target plane, in which blood vessel was to be positioned. The dimension of the line beam was $0.5 \mathrm{~mm}$ by $10 \mathrm{~mm}$. A beam dump was positioned at the downstream in order to block the transmitted beam. The arteries transected from porcine were sandwiched between two transparent slide glasses were placed at or near the focal plane of the cylindrical lens.

In order to find the optimal condition for the sealing of the blood vessels, we placed the vessels at the focal plane of the cylindrical lens (Focus mode) and at the location slighted away from the focal plane of the lens (Defocus mode). Depending on the distance from the focal plane, we could control the size of the line beam in its shorter axis and control the laser irradiation power per unit area. In short, the laser was irradiated to the compressed vessel with the laser power from 5 to $30 \mathrm{~W}$, irradiation time for 5 or 10 seconds, and focus or defocus mode.

\section{Histologic evaluation}

The irradiated arteries were fixed in $4 \%$ formaldehyde and processed to paraffin block. The paraffinized samples were longitudinally sectioned along a long axis of the vessels and stained with hematoxylin and eosin for histological evaluation. After mounted using mounting medium, slides were examined with an Olympus BX51 microscope. Pictures were captured and controlled in Olympus DP72 and DP2-BSW. 


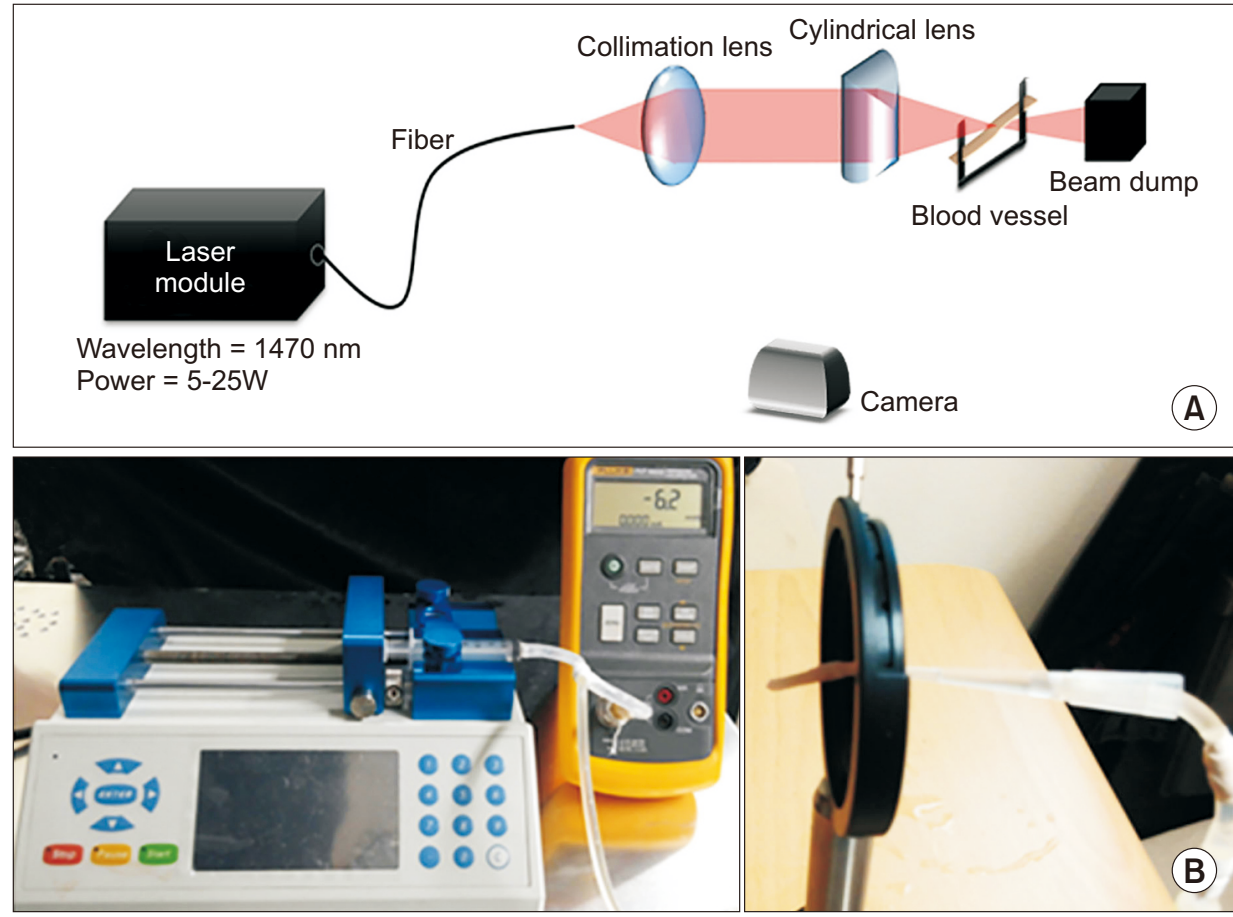

Fig. 1. Experimental setup and burst pressure measurement. (A) Bench-top setup for testing the laser sealing and cutting of blood vessels. 1,470 nm diode laser, collimation lens, and cylindrical lens were used to make a line beam and the arteries were sandwiched between two transparent slide glasses. Subsequently, the line beam was irradiated to the compressed vessel with the laser power from 5 to $30 \mathrm{~W}$, irradiation time for 5 or 10 seconds, and focus or defocus mode. (B) After sealing the vessels, one end of the vessel lumen was placed over a cannula attached to the infusion pump and was closed with iris clamp to seal the vessel onto the cannula. Using the syringe pump, phosphate buffered saline was fed into the vessel and the pressure was measured with the pressure meter. The maximum pressure ( $\mathrm{mmHg}$ ) just before the vessel seal bursts was regarded as the burst pressure for a given sealing condition.

The adequate condition of sealed vessels was defined as showing the even and whole denaturation from the front to back side of the vessels and both intimae were tightly stuck together without any gaps. In addition, the lateral thermal damage zone was defined from 1 to $2 \mathrm{~mm}$ as an adequate condition. However, when occurring an inadequate and localized denaturation of vessels or tissue destruction and detachment by severe thermal damage of vessels, we classified it as an inadequate condition.

\section{Burst pressure measurements}

We measured burst pressure of the sealed vessels to compare the sealing completeness of vessels according to the various sealing condition. Following the standard pressure measurement protocol conducted elsewhere, ${ }^{12,13}$ we constructed a setup composed of pressure calibrator (model 717-100G; Fluke, Everett, WA, USA), syringe pump (model Fusion 100; Chemyx, TX, USA), and Iris clamp (Fig. 1B).

After sealing the vessels, the mid-portions of those were divided with surgical scalpel and then the one end of the vessel lumen was placed over a cannula attached to the infusion pump and was closed with iris clamp to seal the vessel onto the cannula. Using the syringe pump, PBS was fed into the vessel at a flow rate of $7.5 \mathrm{ml} / \mathrm{min}$ and the pressure was measured with the pressure meter. The maximum pressure $(\mathrm{mmHg}$ ) just before the vessel seal bursts was regarded as the burst pressure for a given sealing condition. For control, the burst pressures were also estimated in the vessels sealed with Harmonic Scalpel $^{\circledR}$ and LigaSure ${ }^{\circledR}$ Vessel Sealing System.

\section{RESULTS}

\section{Gross images of the irradiated vessels}

The blood vessels irradiated with 1,470 $\mathrm{nm}$ diode laser were shown in Fig. 2. The laser-irradiated regions were visible in all conditions and showed a tendency to be more charred as the power and the irradiating duration of laser increased. In particular, the char formation tended to increase when irradiating the laser with focus mode and the tissue destruction extensively occurred at an irradi- 

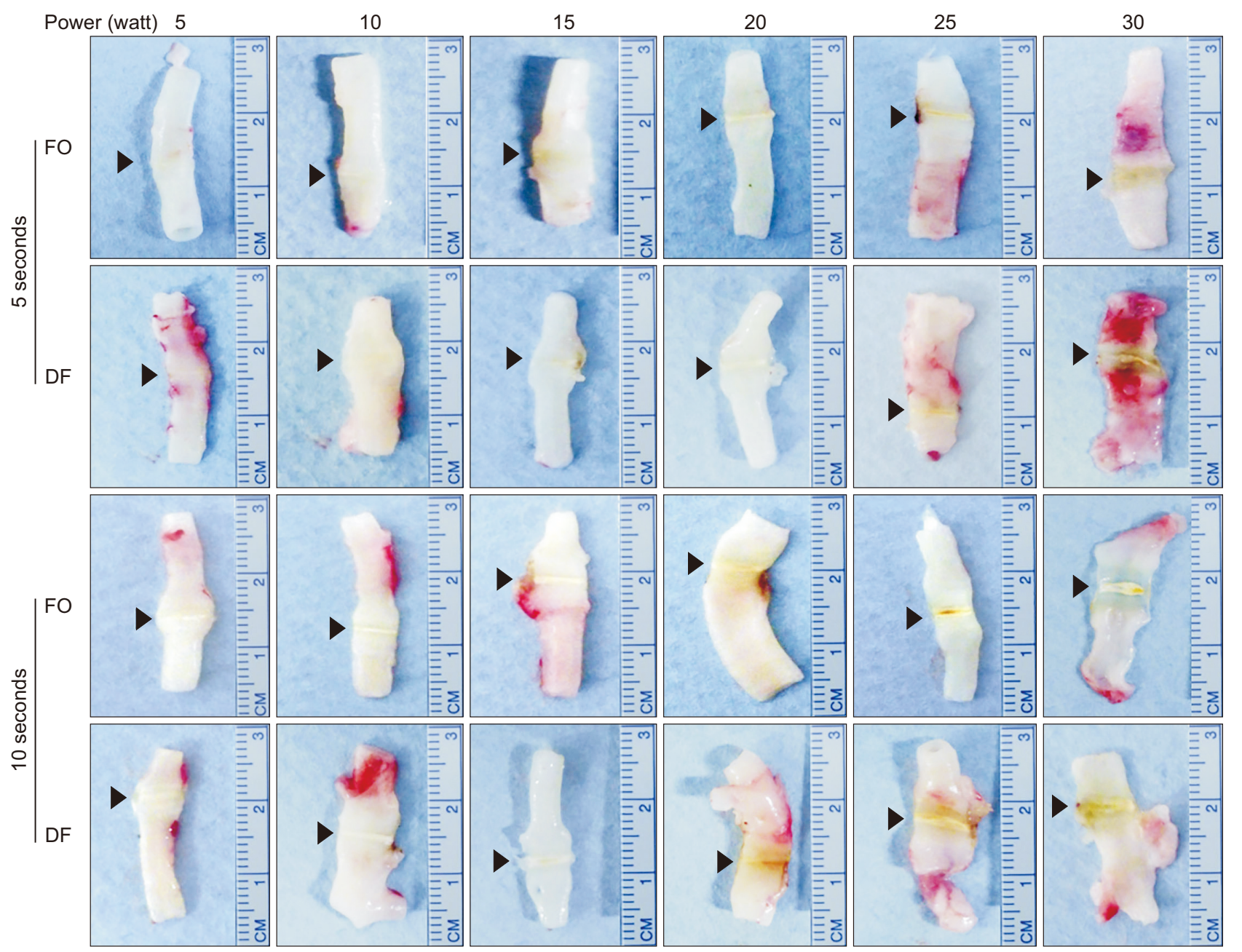

Fig. 2. Gross findings of blood vessels irradiated with laser. The laser-irradiated regions showed a tendency to be more charred in proportion to the power and duration of laser irradiation (arrow head). The tissue destruction extensively occurred at an irradiance of $30 \mathrm{~W}$ for 5 or 10 seconds.

ance of $30 \mathrm{~W}$ for 5 or 10 seconds.

\section{Histologic images of the irradiated vessels}

The histologic findings of the irradiated vessels showed tissue denaturation and adhesion between the front and back side of vessels (Fig. 3). In terms of the completeness of tissue denaturation between the front and back side of vessels, defocus mode was more effective than focus mode, in which the laser was not effectively penetrated into the vessels and the tissue denaturation was not elicited evenly at the full-thickness of the vessels. The vessel walls were relatively more destructed when exposed to the laser irradiation with increasing power from $25 \mathrm{~W}$. In addition, the proportion of denaturation was correlated with the exposure time of laser. When exposed for $10 \mathrm{sec}-$ onds, the irradiated vessels showed relatively wider tissue denaturation than those for 5 seconds. As a result, the adequate condition with the full-thickness and uniform denaturation and lateral thermal damage of the vessel wall less than $1 \mathrm{~mm}$ was the defocus mode laser with an irradiance of $20 \mathrm{~W}$ for 5 seconds.

\section{Burst pressure of the sealed vessels}

Among various conditions of vessel sealing with the laser, we chose the eight conditions for estimating burst pressure; focus mode at irradiance ranging from 15 to 25 W for 5 seconds, defocus mode from 15 to $25 \mathrm{~W}$ for 5 seconds and focus and defocus modes at irradiance of $20 \mathrm{~W}$ for 10 seconds.

The mean of burst pressures, after sealing the vessels with Harmonic Scalpe ${ }^{\circledR}$ and LigaSure ${ }^{\circledR}$ Vessel Sealing System, were from $380 \mathrm{mmHg}$ to $507 \mathrm{mmHg}$ and $410 \mathrm{mmHg}$, respectively. Therefore, the adequate burst pressure was defined as the range between 380 and 507 $\mathrm{mmHg}$ of the control sealing, since it is enough to endure the blood pressure exceeding the normal systolic blood 


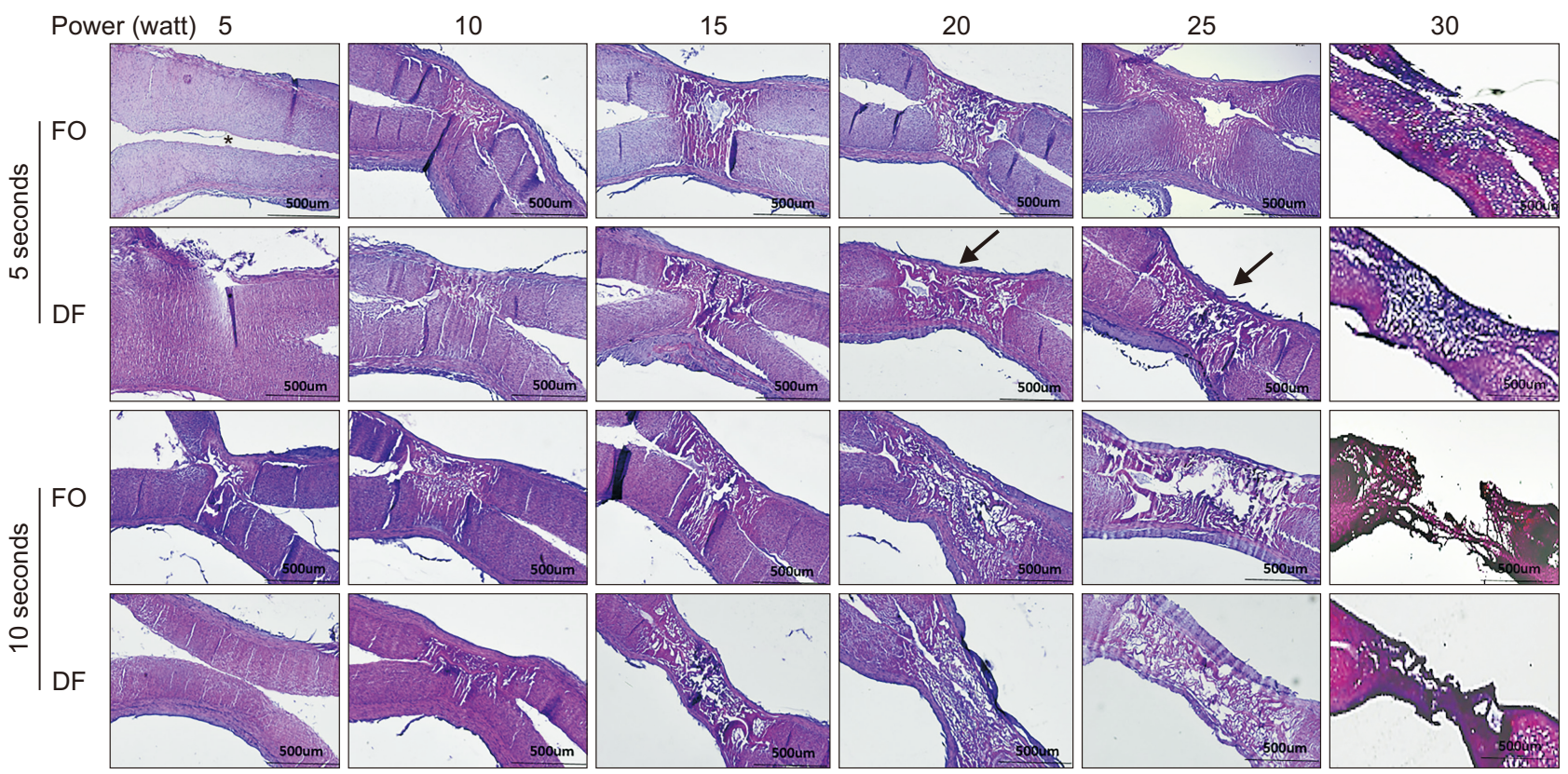

Fig. 3. Histologic findings of blood vessels irradiated with laser. In terms of the completeness of tissue denaturation between the front and back side of vessels, defocus mode was more effective and showed tissue denaturation evenly at the full-thickness of the vessels. The adequate condition with the full-thickness and uniform denaturation and lateral thermal damage of the vessel wall less than $1 \mathrm{~mm}$ was the defocus mode laser with an irradiance of $20 \mathrm{~W}$ for 5 seconds.

pressure of $120 \mathrm{mmHg}$ as well as that above $180 \mathrm{mmHg}$ typically observed in malignant hypertension. Additionally, the other criterion was low standard deviation less than $100 \mathrm{mmHg}$ meaning a consistent sealing of vessels. Consequently, defocus mode at an irradiance of $20 \mathrm{~W}$ for 5 and 10 seconds was the target condition to reveal a sufficient burst pressure (Fig. 4).

\section{DISCUSSION}

The present study demonstrated the adequate condition of laser for vessel sealing through histologic evaluation of blood vessels sealed by irradiation of 1,470 nm near-infrared (NIR) lasers. Comparing to the previous other vessel sealing systems, the sealed vessels under the conditions of defocus mode, $20 \mathrm{~W}$, and irradiation time of 5 to 10 seconds elicited acceptably high burst pressure.

The previous study has suggested that NIR lasers with wavelengths producing optical penetration depths of approximately 0.3 to $0.6 \mathrm{~mm}$ could produce sufficiently strong sealing of blood vessels. In addition, among various wavelengths of laser, 1,470 nm diode laser elicited the ability to fuse vessels ranging from 1 to $6 \mathrm{~mm}$ in diameter with minimal charring and collateral thermal damage at irradiance of $31 \mathrm{~W}$ for a relatively short period less than 5 seconds. ${ }^{10}$ However, in contrast with this previous study, the power of $30 \mathrm{~W}$ showed the excessive destruction of vessel walls in the histologic evaluation of the sealed vessels and the adequate condition for laser sealing system was $20 \mathrm{~W}$, defocus mode, and 5 or 10 seconds of irradiating duration in our study. Especially, the completeness and uniformity of tissue denaturation of the front and back side of blood vessels seemed to be more associated with focus/defocus mode than laser power. Higher power of laser tends to occur more extensive tissue destruction compared to uniform tissue denaturation that ensuring a vessel sealing. Actually, In the present study, laser with focus mode or high power was not effectively penetrated into the vessels and the tissue denaturation was not elicited evenly at the full-thickness of the vessels. However, these results of our study may be associated with an impractical situation, which the laser was irradiated on the collapsed blood vessels sandwiched between two transparent slide glasses. In an actual surgical situation using energy-based devices, blood vessels are collapsed by two end-tips of the device and then cut under a slight tension. Therefore, if the compression pressure to collapse blood vessels increase and if the force pulling on both sides of the vessels is applied, the sealing and cutting of blood vessels may be elicited more effectively and easily.

In conclusion, the extent of tissue change was positively correlated to duration and power of laser. The adequate 


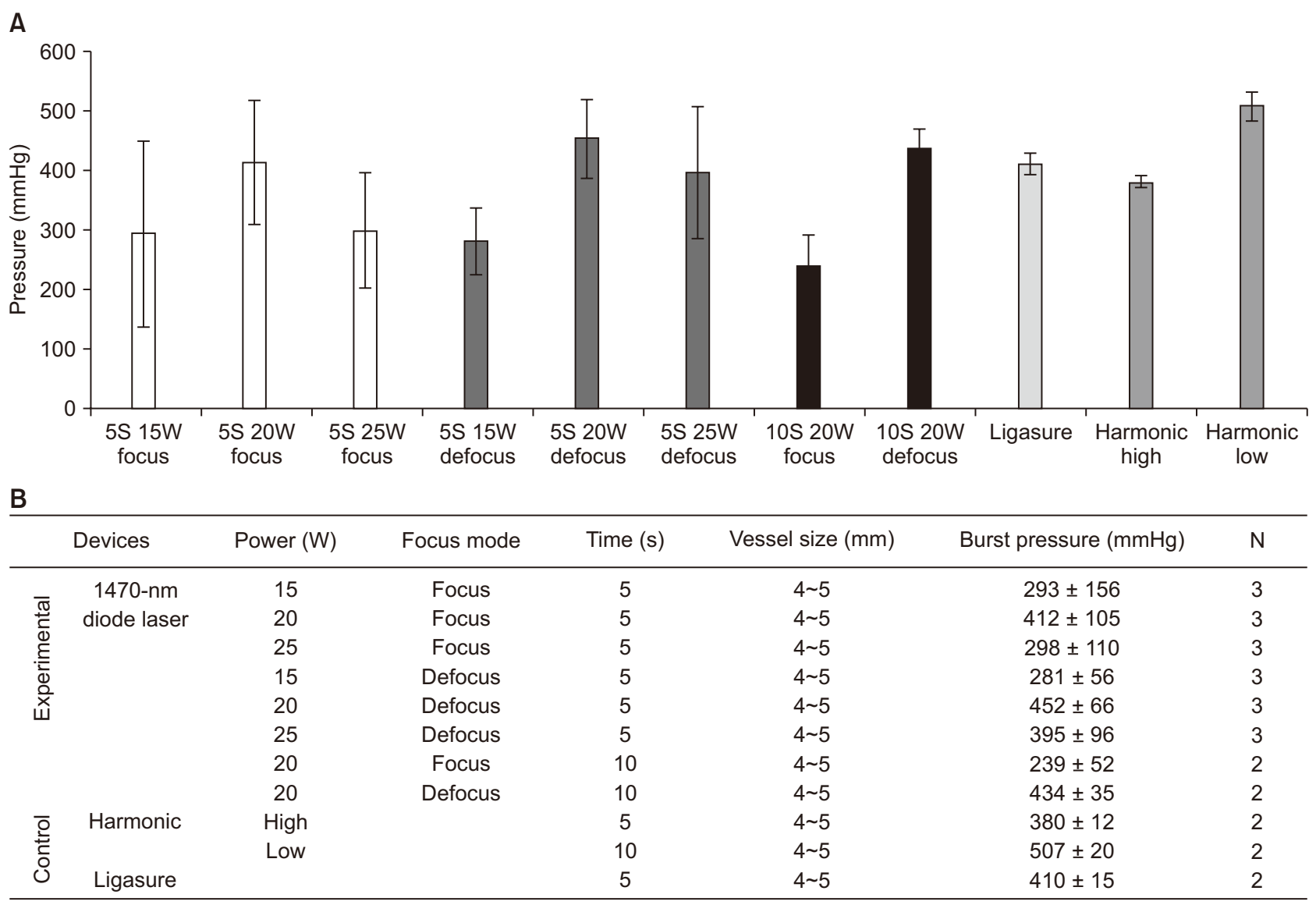

Fig. 4. Burst pressure of blood vessels irradiated with laser. The graph and table show a comparison of the burst pressures of the blood vessels irradiated with laser and those sealed with Harmonic Scalpe ${ }^{\circledR}$ and LigaSure ${ }^{\circledR}$ Vessel Sealing System. When the adequate burst pressure was defined as the range between 380 and $507 \mathrm{mmHg}$ of the control sealing, defocus mode at an irradiance of $20 \mathrm{~W}$ for 5 and 10 seconds was the target condition to reveal a sufficient burst pressure.

condition of 1,470 nm laser for sealing of blood vessels was the power between 15 and $20 \mathrm{~W}$ and exposure time for 5 seconds in defocused mode. Even if the higher power of laser may be required to cut the vessels, the adequate power and irradiation duration of laser can render blood vessels to be sealed effectively.

\section{ACKNOWLEDGEMENTS}

This research was supported by the Korea Health Technology R\&D Project (HI14C0748) through the Korea Health Industry Development Institute (KHIDI) by the Ministry of Health \& Welfare, and the Basic Science Research Program through the National Research Foundation of Korea funded by the Ministry of Education (NRF2016R1D1A1A02937362).

\section{REFERENCES}

1. Kennedy JS, Stranahan PL, Taylor KD, Chandler JG. Highburst-strength, feedback-controlled bipolar vessel sealing. Surg Endosc 1998;12:876-8.

2. Payne JH Jr. Ultrasonic dissection. Surg Endosc 1994;8:416-8.

3. Person B, Vivas DA, Ruiz D, Talcott M, Coad JE, Wexner SD. Comparison of four energy-based vascular sealing and cutting instruments: a porcine model. Surg Endosc 2008;22:534-8.

4. Levy B, Emery L. Randomized trial of suture versus electrosurgical bipolar vessel sealing in vaginal hysterectomy. Obstet Gynecol 2003;102:147-51.

5. Marcello PW, Roberts PL, Rusin LC, Holubkov R, Schoetz DJ. Vascular pedicle ligation techniques during laparoscopic colectomy. A prospective randomized trial. Surg Endosc 2006;20:263-9.

6. Romano F, Gelmini R, Caprotti R, Andreotti A, Guaglio M, Franzoni $\mathrm{C}$, et al. Laparoscopic splenectomy: ligasure versus EndoGIA: a comparative study. J Laparoendosc Adv Surg Tech 
A 2007;17:763-7.

7. Kim FJ, Chammas MF Jr, Gewehr E, Morihisa M, Caldas F, Hayacibara E, et al. Temperature safety profile of laparoscopic devices: Harmonic ACE (ACE), Ligasure V (LV), and plasma trisector (PT). Surg Endosc 2008;22:1464-9.

8. Phillips CK, Hruby GW, Durak E, Lehman DS, Humphrey PA, Mansukhani MM, et al. Tissue response to surgical energy devices. Urology 2008;71:744-8.

9. Govekar HR, Robinson TN, Stiegmann GV, McGreevy FT. Residual heat of laparoscopic energy devices: how long must the surgeon wait to touch additional tissue? Surg Endosc 2011;25:3499-502.

10. Cilip CM, Rosenbury SB, Giglio N, Hutchens TC, Schweinsberger GR, Kerr D, et al. Infrared laser thermal fusion of blood vessels: preliminary ex vivo tissue studies. J Biomed Opt 2013;18:58001.

11. Giglio NC, Hutchens TC, Perkins WC, Latimer C, Ward A, Nau $W H$, et al. Rapid sealing and cutting of porcine blood vessels, ex vivo, using a high-power, 1470-nm diode laser. J Biomed Opt 2014; 19:38002.

12. Hope WW, Padma S, Newcomb WL, Schmelzer TM, Heath JJ, Lincourt AE, et al. An evaluation of electrosurgical vesselsealing devices in biliary tract surgery in a porcine model. HPB (Oxford) 2010;12:703-8.

13. Newcomb WL, Hope WW, Schmelzer TM, Heath JJ, Norton HJ, Lincourt AE, et al. Comparison of blood vessel sealing among new electrosurgical and ultrasonic devices. Surg Endosc 2009;23:90-6. 